\title{
Development
}

\section{Partnerships for skills training in the care-home sector}

\author{
Julie Cooper ${ }^{1}$, Anne Levington ${ }^{2}$, Stephen Abbott ${ }^{3}$ and Julienne Meyer ${ }^{4}$ \\ ${ }^{1}$ Senior Research Fellow, Care for Older People (Nursing Homes), School of Community and Health Sciences, City \\ University London, London, UK \\ ${ }^{2}$ Practice Facilitator, Barts and the London NHS Trust, London, UK \\ ${ }^{3}$ Research Fellow, School of Community and Health Sciences, City University London, London, UK \\ ${ }^{4}$ Professor, Care for Older People, School of Community and Health Sciences, City University London, London, UK
}

\begin{abstract}
Aim: This paper describes an initiative in North East London that aimed to facilitate access to training for care-home staff by using a mobile skills-centre in the form of an adapted bus. Background: It has proved difficult to take a strategic approach to quality assurance in care homes and the first comprehensive national training strategy for the sector was not published until 2000. Staff value and benefit from training, but organizing the provision of education and training may be problematic, given resource constraints and staffing levels that make it difficult to release staff to go off-site. Method: Collaboration between the School of Community and Health Sciences, City University London; My Home Life, an initiative led by Help the Aged in collaboration with the National Care Forum and City University London; local care homes; local primary care trusts (PCTs); and the Centre for Excellence in Teaching and Learning at City University London and Queen Mary University of London. The project involved facilitation, training in the mobile skills-centre and evaluation through questionnaires. Findings: The project was successful at a number of different levels: providing training to care-home staff; fostering collaborative relationships between care homes and PCTs; providing a forum to enable a wider educational discussion of care-home needs; and stimulating the planning of future education programmes for care-home staff and of the provision in care homes of student nurse placements.
\end{abstract}

Key words: care homes; partnerships; skills training

Received 22 April 2009; accepted 11 July 2009; first published online 14 August 2009

\section{Introduction}

The role of care homes in providing communitybased care for people who cannot, even with high levels of domiciliary health and social care, live independently, has long been recognized (Kirkwood and Astle, 1996; Dudman, 2007). Care homes in England are very diverse, and include both large

Correspondence to: Stephen Abbott, Research Fellow, School of Community and Health Sciences, City University London, 20 Bartholomew Close, London EC1A 7QN, UK. Email: s.j.abbott@city.ac.uk

(C) 2009 Cambridge University Press and small organizations in the public, private and not-for-profit sectors. This diversity is one reason why it has proved difficult to take a strategic approach to quality assurance in care homes: it is only recently that attempts have been made to introduce standards-setting and regulation at a national level (the Care Standards Act, 2000).

By the nature of their frailty, disability or illness, care-home residents are likely to be significant users of NHS services. Because they are smaller than general hospitals, care homes cannot always provide specialist care, and even those that offer nursing care are unlikely to have many 
registered nurses on duty at any one time. This may result in 'unnecessary' demand being placed on NHS services; 'unnecessary' because the care required could be provided within the care home, were there the capacity and expertise to do so. In the absence of such capacity and expertise, care homes may call in the general practitioner or district nurse, for example, for blood tests or diabetes management; or they may send residents to Accident and Emergency departments with urgent minor problems, for example, a blocked catheter. It would be preferable both from the point of view of optimal use of services and from the point of view of the convenience of residents (and staff) if such care could be provided in the homes. There is thus a clear incentive to ensure that training programmes are available to support and increase the skills of staff, although the first comprehensive national training strategy for the sector was not published until 2000 (TOPSS, 2000). Needs assessment at a national level is difficult because of lack of data about staffing and skill-mix levels (Dudman, 2007), and little is known about the reality of education and training (Meyer, 2007). Staff value and benefit from training (Nolan and Keady, 1996; Clelland et al., 2005), but organizing the provision of education and training may be problematic, given resource constraints and staffing levels that make it difficult to release staff to go off-site. For this reason, Meyer (2007) suggests that

creative learning initiatives delivered in the workplace appear to be the way forward.

(2007: 134)

This paper describes such an initiative that was carried out in two boroughs in North East London in 2008. The initiative aimed to facilitate access to training for care-home staff by using a mobile skillscentre in the form of an adapted bus. It was also recognized that the project had the potential to actively engage care homes in the joint planning of training in a way that had not previously been achieved. The paper begins by identifying the project's stakeholders and briefly describing the bus. It then gives an account of the initiative's development and outcomes, and highlights transferable learning.

This report is based on data derived from five sources:

- participant observation of a variety of planning meetings;
- official minutes of these and other meetings;

- two one-to-one interviews with care-home managers;

- feedback forms completed by care-home staff attending teaching sessions on the bus;

- feedback forms completed by staff that taught sessions on the bus.

\section{The stakeholders}

The project represents the coming together of a range of organizations and organizational agendas:

- The School of Community and Health Sciences (SCHS), City University London. SCHS provides education to students of a range of health professions, and to health professionals working in East London, and seeks to arrange placements for nursing students in local care homes.

- My Home Life (MHL). MHL is an initiative led by Help the Aged in collaboration with the National Care Forum and City University London, which seeks to improve the quality of life of those living, dying, visiting and working in care homes for older people.

- Care homes. These are owned and managed by a variety of private and not-for-profit national and local organizations, and provide care for, among others, clients of local Social Services Departments and patients of local Primary Care Trusts (PCTs).

- PCTs. PCTs purchase care from local care homes for patients with continuing health care needs, and offer support to care homes and their residents from their own specialist nursing staff. They work closely with SCHS to provide placements for student nurses.

- The Centre for Excellence in Teaching and Learning (CETL) at City University London and Queen Mary University of London. This collaboration seeks to enhance the student experience by increasing the teaching and learning resources available, with emphases on clinical and communication skills, and interprofessional learning.

In keeping with the principles of MHL, it was crucial that this was not simply a SCHS initiative, but a genuine partnership with care homes, PCTs and MHL. 


\section{The bus}

The clinical skills bus (Nicol et al., 2007) is a mobile skills-centre equipped with equipment to support learning about a range of core skills such as:

- temperature, pulse and blood pressure recording;

- taking blood samples and inserting drips;

- cardiac resuscitation skills;

- physical examination and assessment;

- blood glucose monitoring;

- catheterization.

A laptop and a data projector are also available. There is a full-time bus driver who is also a clinical skills facilitator, and another clinical skills facilitator can act as relief driver when needed. Those arranging to use the bus (university departments, NHS organizations, care homes, and so on) can provide their own teachers.

Although buses have been used to make health promotion resources more accessible to the public (Wild, 2007; Logan, 2008), it is less usual to use them for training purposes.

\section{Developing the project}

The project plan was based on an acknowledgement that care homes often find it difficult to send staff to off-site training, being limited by staffing levels, training budgets and travelling distances. By offering sessions on the bus, it was hoped that care homes would find access easier, and as an additional incentive, no charge was made for either the use of the bus or the training provided as part of this pilot project. Members of the project team therefore sought to facilitate discussions between the care homes and the PCT in each borough to identify training needs that would then be met by using the skills bus, with SCHS or NHS staff leading the sessions. It was also hoped that the project would plant the seeds for future collaboration, to develop ongoing training and education programmes. Participation in the project was voluntary, and nine out of a possible ten homes took part: in one borough, all three homes sent staff, while in the other, six out of seven did so. All the participating homes were care homes with nursing. Their capacity (number of beds) ranged from 43 to 120 .
For a number of reasons, the process of setting up the project was slow. An existing forum for care homes and the PCT met regularly in one borough, but it already had a busy agenda. It was not possible to organize additional meetings that all the care homes could attend, and it was thus hard to find sufficient time to include planning for the bus project; the process took several months longer than originally expected. This was also true in the other borough, where such a forum did not already exist, although some individual PCT personnel did have good links with care homes. Also, the PCT had recently created a new post of Clinical Nurse Specialist to work specifically with care homes, and it was felt that the holder of the new post would ideally be closely involved in the initiative. In the event, design of the post and recruitment to it suffered a number of delays, and the post-holder arrived when planning for this project was virtually complete.

In keeping with the MHL theme of sharing decision-making, the managers of the care homes were invited to draw up a list of training topics that they and their staff would value. Facilitating the links, arranging meetings and acting on the resulting decisions by coordinating the bus, carehome staff and teachers was extremely complex and time-consuming for project team members.

\section{Delivering the project}

In all, 15 half-day training sessions were arranged, and were attended by about 100 staff altogether (the number of those attending one session were not recorded exactly, and some staff attended more than one session). These were held on the bus, which was parked outside one of the participating care homes, between April and September 2008. Topics were:

- Continence, catheter care, and catheterization (four sessions);

- Venepuncture (two sessions);

- Falls prevention (two sessions);

- Diabetes (two sessions);

- Epilepsy (one session);

- Acute assessment skills (myocardial infarction, transient ischaemic attacks and stroke) (one session);

- Nutrition, swallowing and feeding (one session); 
- Dementia and challenging behaviour (one session);

- Record-keeping and actioning care (one session).

Of these, the venepuncture, epilepsy and acute assessment skills sessions were open to registered nursing staff only, but the remainder were attended by both registered nurses and care assistants. Teaching staff were drawn from the PCTs, SCHS, and local hospitals.

It will be noted that the sessions largely focused on clinical skills. This reflects the findings of Morrell et al. (1995) that care-homes staff tend to prioritize clinical nursing issues over issues relating to quality of life. During the planning period, SCHS staff expressed a readiness to offer sessions relating to quality of life, but care-home managers, while acknowledging the importance of these, felt that deficits in clinical skills were more pressing. Also, care homes do not necessarily require outside trainers to address quality of life issues, but may do in the case of some clinical skills.

\section{Results of the evaluation}

Those attending were asked to complete a short evaluation sheet as each session came to a close (this was omitted in one session). Of the 88 received, 60 were from registered nurses and 24 from care assistants (in four cases, staff did not identify their role). Some of these may have been completed by the same staff after different sessions, but because forms were anonymous, this is unclear. Analysis of these showed that students were virtually unanimous in their praise of the content and presentation of the sessions, and they generally found the bus an interesting and enjoyable environment, conducive to learning. In 16 cases, there were some mild criticisms of the bus, such as that the bus was small, stuffy, too hot or cold, unsteady or equipped with uncomfortable chairs. However, there is plenty of anecdotal evidence that comparable criticisms are frequently made by students and teachers about SCHS classrooms and PCT training rooms.

Feedback was also received from all 10 teachers, using a different evaluation form, who rated the bus as a good or very good teaching environment. Though most of them had little or no experience of teaching care-home staff, the knowledge, skills and learning needs of the students, as attended, were generally as expected. Some teachers remarked to project leads that they were impressed by the staff attending, their enthusiasm to learn and their knowledge levels ('it was a joy to teach them'). This suggests that it is confidence rather than knowledge that staff primarily lack, and that external perceptions of care-home staff as under-skilled may be misjudged. However, more work is needed to support or disprove this interpretation.

There were also three significant longer-term outcomes:

- In one borough, the project put the care homes in touch with each other, and there was evidence at project meetings of information exchange and joint problem-solving about matters unrelated to the project itself. The project also promoted relationships between the care homes, the PCT and SCHS: there are plans to develop an ongoing forum.

- Discussions have begun about arranging for some care homes in both boroughs to offer student nurse placements. It had previously been difficult to initiate such discussions.

- SCHS is planning to develop ongoing training for the care-home sector. This will consist of a rolling programme of short courses about clinical skills, and a foundation degree course focusing on quality aspects of care.

\section{Challenges}

There were a number of challenges that had to be met, which one can predict would be encountered in similar projects. First, coordination of the project was complex and time-consuming, and carried out by project team members in addition to their everyday work. This reflects the fact that there are numerous care homes, representing a variety of organizations (small, large, independent, part of a large chain). Communications are thus much more complicated than when education is being negotiated with local PCTs. Similarly, care homes vary considerably in their needs. Some find it easier to release staff for a whole day; others prefer shorter periods. Some can draw on extensive in-house training provided by their parent organization; others are independent. The size of training budgets varies. One result of this diversity was that it took some time to develop consensus 
about what training should be provided. Diversity self-evidently precludes the suitability of a 'one size fits all' model of education provision, though it has to be recognized that more flexible and tailor-made models tend to be more expensive because they lack economies of scale.

Training in some clinical skills requires follow-up sessions to assess and ratify individuals' competence. The project did not set out to provide these, and it became evident that care homes and PCTs did not find it easy to make suitable arrangements for them. In some care homes, a trained member of the staff might be able to make some of these assessments. But in other cases, external staff with appropriate expertise was needed. Although suitable NHS staff was identified, they sometimes found it difficult to make time for this additional task, while in one case, a hospital charged for the service. In this case, the project was able to provide the money required, though this cost had not been allowed for in the budget.

A potential challenge arises from the fact that the role of PCTs in relation to care homes is not straightforward. While PCTs wish to commission high-quality care for their patients, it is not their responsibility to support that quality by providing or commissioning care-home staff training. In both the PCTs in this project, there was strong senior commitment to offering care homes support, and so this was not a stumbling block, but it cannot be assumed that this would always be the case elsewhere. Public sector personnel are not always fully accepting of the 'mixed economy of care' and can be suspicious of for-profit organizations, and negative attitudes to the care-home sector were very occasionally encountered at a less senior level during this project.

\section{Conclusion}

Thus, the project has been successful at a number of different levels:

- providing training to some care-home staff;

- fostering collaborative relationships between care homes and PCTs in two boroughs;

- providing a forum to enable a wider educational discussion of care-home needs;

- stimulating the planning of future education programmes for care-home staff, and of the provision in care homes of student nurse placements;

- affirming the principles of MHL: creating community, sharing decision-making, improving healthcare, keeping the workforce fit for purpose, and promoting a positive culture (by acknowledging the value of the sector, and offering support).

Although CETL made available some resources for evaluation of the initiative, it was not possible to investigate important questions such as the project's benefits to patient care or to staff satisfaction. Nor were we able to measure change in the skills and knowledge of those attending the training. Thus, we cannot document the project's contribution to quality of life in care homes. But we can point to some learning that may be transferable to other areas.

First, adopting a bottom-up approach (ie, establishing good links with care-home managers and allowing them to determine the content of the training sessions) was crucial to the philosophy of the project, but, like the organization of the sessions themselves, it was very labour-intensive. Teaching staff cannot be expected to carry out such administrative functions on a continuing basis, as they did for the duration of this project: administrative support must be funded. Indeed, the administrative function would have to be expanded to include more boroughs in order to create a sufficiently large market for ongoing educational provision. Relevant costs would have to be reflected in charges for training, thus becoming a potential barrier to access for homes with very limited training budgets.

Second, as already indicated, it is important to ensure that the focus of training is not taken to be purely clinical. In addition to health care training, other evidence suggests that homes need educational support to ensure that their staff's interactions with patients are sensitive and understanding (Henwood, 2001). Quality of life issues were very important to the project team, which was able to influence the planning of an ongoing education programme for the care-home sector by SCHS, which, it is intended, will include a foundation degree based on quality of life issues. But this emphasis came from educators rather than from care homes: the bottom-up approach may not guarantee such an emphasis, and so educators may need to offer leadership in this area. 
Third, the project was an experiment. Its title was 'Meeting the training needs of the care-home sector: Can the CETL bus help?', and it was undertaken very much in a spirit of 'seeing what happens'. Thus, it was typical of other creative learning initiatives in that it was

\section{driven by enthusiasm and hampered by short-term funding.}

(Meyer, 2007: 134)

Short-term funding creates problems of sustainability, but it was probably an appropriate method for making possible an experiment such as this, where the outcome was uncertain. What is essential is that the learning for the medium- and long-term is extracted from the short-term project, and that mechanisms are found for more enduring funding arrangements (something that we have not yet achieved, though discussions are continuing).

Fourth, the project took advantage of a locally available resource, the bus, for which it did not have to pay. It is interesting to speculate whether these outcomes could have been achieved without the use of the bus. A considerable proportion of the teaching provided in the project did not strictly speaking require the bus, as it did not require specific clinical equipment. Indeed, the bus is primarily suitable for skills sessions rather than lectures or discussion, as the long thin shape means that sightlines can be poor, and groups cannot sit in a circle. Thus, some sessions could have been carried out wholly or partly in care homes' own training rooms, although these often lack presentation facilities such as PowerPoint. On the other hand, there is no doubt that using the bus encouraged all parties to think of education provision in new ways, and that the easier access it offered encouraged care homes to release staff for learning. The bus was thus a catalyst for important developments. In itself, it is unlikely to be an essential component in future education provision within the care-home sector, though it will continue to be useful. Its value was to stimulate interest and engagement and it has demonstrated that, though the care-home sector is regarded as somewhat 'hard to reach' for both the NHS and for educational institutions, innovative approaches can create productive engagement.

\section{Acknowledgements}

We are grateful to the Centre for Excellence in Teaching and Learning, City University London and Queen Mary London University, for making the clinical skills bus available, and funding SA's time.

\section{References}

Care Standards Act. 2000: Available at www.opsi.gov.uk/acts/ acts2000/ukpga_20000014_en_1

Clelland, J., Scott, D. and McKenzie, D. 2005: An analysis of allied health professional training in care homes for older people in Glasgow. Quality in Ageing - Policy, Practice and Research 6, 24-36.

Dudman, J. 2007: The context. Ch. 1. In National Care Homes R\&D Forum: My Home Life, Quality of life in care homes. A review of the literature. London: Help the Aged, 5-17. Available at http://www.myhomelife.org.uk/resources/ mhl_review.pdf

Henwood, M. 2001: Future imperfect: report of the King's Fund Care and Support Worker Inquiry. London: King's Fund.

Kirkwood, A. and Astle, J. 1996: Long-term care: a framework for reform. London: Institute of Community Studies.

Logan, L. 2008: Improving uptake of cervical smear tests. Independent Nurse, 45-47.

Meyer, J. 2007: Keeping the workforce fit for purpose. Ch. 10. In National Care Homes R\&D Forum: My Home Life, Quality of life in care homes. A review of the literature. London: Help the Aged, 124-41. Available at http:// www.myhomelife.org.uk/resources/mhl_review.pdf

Morrell, J., Mernick, M., Brown, R. and Brooker, C. 1995: The training needs assessment of qualified nurses in private nursing homes in Trent. Sheffield: School of Health and Related Research, University of Sheffield.

Nicol, M., Warren, A. and Connolly, J. 2007: Development of a clinical skills bus: making simulation mobile. International Journal of Clinical Skills 1, 101-13.

Nolan, M. and Keady, J. 1996: Training in long-term care: the road to better quality. Reviews in Clinical Gerontology 6, $333-42$.

TOPSS (Training Organisation for the Personal Social Services). 2000: Modernising the social care workforce: the first national training strategy for England. London: TOPSS.

Wild, S. 2007: Improving health outcomes for children. Independent Nurse 8, 44-45. 